\title{
A NEW CONTROL FRAMEWORK FOR ROBOT SOCCER SYSTEM
}

\author{
*Dong-Hun Lee, Seung-Min Baek, Tae-Kyung Moon, Chae-Wook Chung, and **Tae-Yong Kuc \\ ICon Lab, School of Information \& Communication Engineering \\ Sung Kyun Kwan University, Suwon 440-746 Korea \\ *neowintop@icon.skku.ac.kr;**tykuc@yurim.skku.ac.kr
}

\begin{abstract}
This paper presents a new control framework for robotic soccer system which consists of a group of 11 micro-robots, external vision system, ultrasonic GPS sensor network, RF communication modules, and main computer to perform a distributed sensing and control for successful soccer game in X-large league Mirosot of FIRA[11]. In the proposed control framework, even though the single CCD camera covers limited area of workspace and each GPS sensor has limited capability with a simple signal-processing engine, combined usage of the two kinds of sensors makes it possible to perform a large task through coordinated information sharing and wireless communication covering a large working area. Using the capability of self-localization and tracking with the sensor network, we show the proposed control framework can be applied to a large scale robot soccer system in which a large number of robots have to be coordinated effectively in real time. Copyright (C) 2005 IFAC
\end{abstract}

Keywords: Robot soccer, sensor network, mini-mobile robot, control architecture, ultrasonic GPS.

\section{INTRODUCTION}

Cooperative robotic behaviors are aiming at achieving some goal in distributed working environment which in general a single robotic behavior or a single operation of robot can not succeed in. The robot soccer is a typical example of task which needs cooperative robotic behaviors of multiple robots to perform successful soccer game and record a 'goal'. When constructing a robotic soccer system, there are two choices one can select for control architecture of multiple mobile robots to generate cooperative behaviors: centralized and decentralized. Centralized control is characterized by a single control agent. For example, Mirosot game category of FIRA[11] provides a centralized control environment with a main computer in charge of reasoning and decision making for coordinated motion generation of multiple soccer robots. The sensing and perception of working environment from the external vision system is also centralized in that no other sensor contributes in global positioning and generation of cooperative behaviors of multiple robots.
Recent advances in hardware and software of soccer robots as well as algorithms for control strategy have made it possible for us to integrate a X-large scale robot soccer system which consists of 11 mobile robots. Since the robot soccer playground in this category is much larger than the usual Mirosot game, the external vision system with a single CCD camera only may be not adequate for complete environment perception due to its limited coverage and resolution. The difficulty is added when the image of ball is occluded by the robots located near the ball. In solving the problems due to the difficulties, since an external camera located at higher than $3 \mathrm{~m}$ is not allowed in the X-large league Mirosot, two options can be considered:

i) Centralized sensing and perception with multiple cameras

ii) Decentralized sensing and perception with distributed heterogeneous sensors

While the first solution gives us a direct extension of the centralized sensing and control strategy used in the Mirosot category, it can be much expensive and needs higher computational power. The second 
solution necessitates a new control framework with distributed sensing and signal processing, since it is completely different from that of robotic soccer system for the usual Mirosot category. In this work, we propose a new control framework for X-large scale robotic soccer system by using a distributed sensing and perception approach with a single camera and simple ultrasonic GPS sensor network.

In general, the sensor network is short-range wireless network consisting of large number of sensor nodes densely deployed with different specs and functions. Each sensor node can contain various sensors, low power processor, wireless transceiver, and limited amount of battery. The sensor node periodically samples environment data and sends processed information when requested from other node. Since sensor network is highly redundant in a small area and processors are highly distributed at each node, many samples of data can be obtained from every single phenomenon resulting in high fault tolerance [2-7]. Moreover, each sensor node can be used as smart beacon to recognize the workspace providing much richer information for localization and navigation of mobile robot than the traditional passive beacon.

In the new control framework, the combined usage of camera and ultrasonic GPS sensor network is complementary. That is, even if the two different sensors have limited capability when operating separately, the synergetic use of the two kinds of heterogeneous sensors makes it possible to perform a large task through coordinated information sharing and wireless communication covering a large working area. Using the capability of distributed sensors for self-localization and tracking of moving objects, we show the proposed control framework can be applied to a X-large scale robot soccer system in which a large number of robots has to be coordinated effectively in real time.

\section{PRELIMINARY}

When generating intelligent robotic behaviors of multiple soccer robots cooperating with one another and competing with the opponent robots in a highly dynamic environment of soccer playing ground, a lot of information has to be gathered using all the possible features extracted from sensed physical data of various sensors. Generally speaking, since in the centralized multiple robotic systems as in the Mirosot category, the vision sensor as well as actuators of robots are wired and fixed in configuration, planning, equipping, and maintaining the systems demand high procedural complexity and cost, which in turn implies difficulties in constructing a decentralized control architecture for distributed sensing and actuation as in the ubiquitous computing environment. More specifically, in the X-large scale robot soccer system, the single usage of $3 \mathrm{~m}$ high fixed external camera and vision data processing system may have limited performance in dexterous real-time coordination of multiple mobile robots due to occlusion of ball close to robot, tradeoff in between computational power and requirement of sufficiently high resolution vision data to identify all the clouded objects in the large work space, etc. The difficulty can be overcome by introducing a wireless sensor network that consists of a group of sensor nodes resulting in a new control framework of decentralized sensing, information processing, and control.

\subsection{Sensor Node}

Sensor node is basic unit of sensor network; consisting of low-power processor, sensor, memory, low-power transceiver and battery. Including sensors detecting temperature, humidity, intensity, acceleration, terrestrial magnetism, infrared from human, sensor node can be used for various applications. Each sensor node interacts with its adjacent nodes in the wireless network by using wireless communication. In the sensor network, each sensor node collects and analyzes sensor input periodically, while performing appropriate tasks as information transmission when requested from other nodes. While a sensor node can communicate using various wireless media such as RF, IR, LASER and ultrasonic, the low power ISM band short range transceiver is commonly used.

\subsection{Sensor Node Localization}

Self-localization of each sensor node is important in sensor network. However, being randomly scattered in the interested area, the manual confirmation of every node position is not plausible or possible. Instead, several reference nodes are placed in the network whose absolute position can be measured. Using these reference nodes, self-localization of all the sensor nodes can be made by measuring relative distances or angles to the adjacent references. In calculating the absolute position of each node, any positioning algorithm like triangular method or ABC (Assumption Based Coordinates) can be used. After obtaining the absolute positions of fixed nodes, relative distances to some moving node like mobile robot can be also calculated by signal arrival time, or by strength of received signal (TOA, TDOA, RSSI). On the other hand, angles between two nodes can be measured by vector pressure sensor or acoustic sensor array (DOA, AOA). More detailed exposition of the sensor localization techniques can be found in the related topics as Active Badge, Active Bat, Cricket [7], RADAR, VOR/VORTAC, and Cellular Networks.

\subsection{Linking and Routing in Sensor Network}

Since the connection of sensor nodes in sensor network needs to be robust for network reliability, linking layer and routing algorithm is one of the most important issues in sensor network research. The Ad Hoc Demand Distance Vector (AODV) and Temporally Ordered Routing Algorithm (TORA) are not optimal because of the low mobility of the system. So, it is more preferable to go with a tablebased system for routing of sensor network. In the method, each sensor updates a table periodically keeping the routing path available to reduce transition overhead, network traffic, and packet losses [2]. Since there are a lot of nodes in the 
relatively small area, the network should be carefully managed and using the global ID such as MAC address is not recommended. Instead, each node needs to be accessed via attribute-based naming rather than explicit addressing, i.e. direct diffusion.

\subsection{Robotics Applications}

Deliberation is traditional planer-based control algorithm but unresponsive and slow to adapt to dynamic environment. Reactive system that has tightly coupled perception and action is responsive but lack of generality and ability to store representation for serving complicated tasks. Modern behaviour-based control generalizes reactive control by introducing the notion of behaviour as an encapsulated time-extended sequence of actions. Perception and response are still coupled as in reactive systems with the added benefits of representation and adaptation without any centralized control. In robot control, as seen in before, sensing and action is tightly coupled and accurate environment recognition should be provided for correct robot responds. Improving a single sensor performance is limited and costs high. Moreover, using few samples from limited number of sensors embedded in robot to estimate the environment is not enough. Combination of mobile robot and sensor network enables the sensor fusion, and many samples obtained from every single phenomenon can increase the measurement accuracy.

\section{A NEW CONTROL FRAMEWORK FOR DISTRIBUTED SENSING AND ACTUATION}

In this section, we describe the entire system and control architecture for mobile robot within the sensor network embedded workspace.

\subsection{System Hardware}

The proposed robotic soccer system consists of 11 $7.5 \times 7.5 \times 7.5 \mathrm{~cm}$ micro robots fit to the X-large league MiroSot specification, an external vision system, a small size ultrasonic GPS sensor network, and a main PC.

\section{A. The Robots}

Two independent DC motors drive each robot and uses encoders for dead reckoning in local navigation, while the supervising central coordinator with the external vision system and sensor network normally guides the global coordination. Our micro mobile robot has on top 16bit microprocessor Intel 80C296SA to provide a fast PID servo loop for two DC motor driven wheels. Each mobile robot is also equipped with a GPS module for self-localization in the sensor network and interaction with other sensor nodes as an active moving node.

\section{B. The Vision System}

In the MiroSot, an external color vision system is used to provide the raw data of planar ground for visual information extraction. The vision system consists of a CCD and frame grabber interfaced with the main computer. The CCD provides NTSC signal output for the frame grabber to convert the raw data, into the digital data at a maximum rate of 60 fields/set. Each pixel in the 514 x 490 array of pixels in the image plane is assigned some RGB values of 8 bits data, which are further processed by the vision data processing algorithm for object recognition.

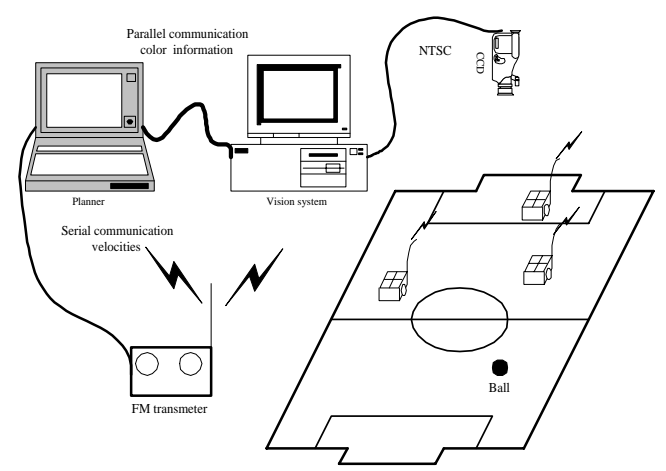

Figure 1. Robotic soccer system

However robot localization should be done in itself in the standalone soccer robot (RoboSot). Applying the sensor network consist of ultrasonic sensor GPS nodes to workspace (game area), this problem can be solved.

\section{The Communication Link}

The IR or RF communication module can be used in transmitting the continuous stream of information data from the centralized coordinator to each robot playing soccer game. In the ordinary robotic soccer system, only the unidirectional communication link is implemented meaning the transmitter is used by the main computer and the receiver is onboard mobile robot. To collaborate the robot with sensor network, the bidirectional wireless communication should be performed.

D. The Sensor Node Hardware

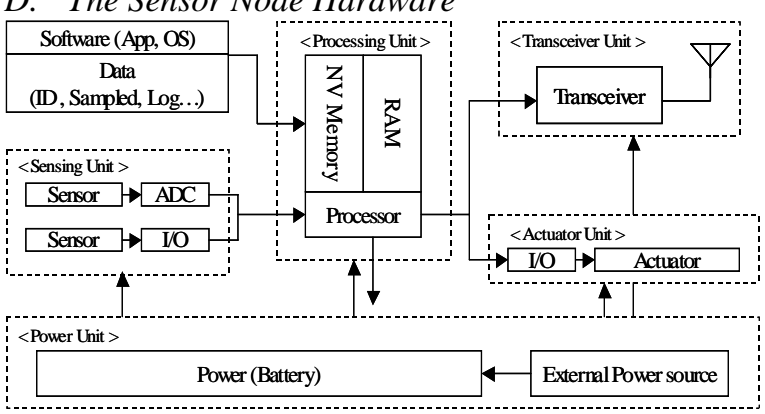

Figure 2. Block diagram of sensor node.

Sensor node is made up of five components, as shown in Fig2: a sensing unit, a processing unit, a transceiver unit, power unit, and actuator unit. The sensing unit consists of sensor and ADC, converts the analogue signals of sensor output to digital data, and passes to the processing unit. NV memory in processing unit contains OS for node management, and binary image of node assigned tasks. Furthermore, this space can be used to store processed data and operation log, so, when the network or node failure occurs, node can restore job after the error recovered. A transceiver unit connects the node to the network and various kinds of media can be used, while for indoor application, short range $\mathrm{RF}$ is recommended. A power unit cuts off the power supply to unused unit and charge internal battery while external power source is available. An actuator 


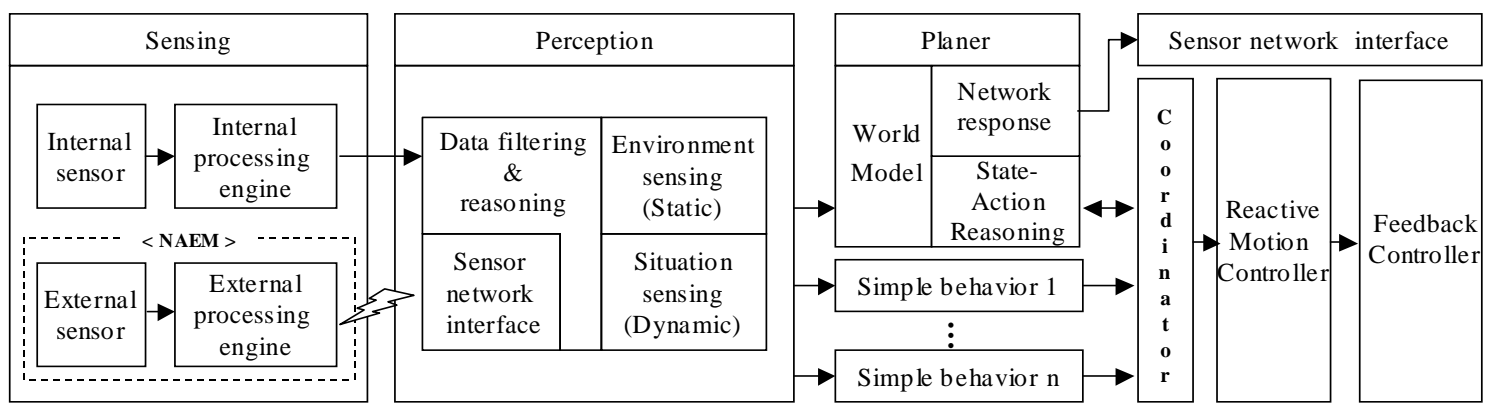

Figure 4. Integrated intelligent control architecture

unit is an optional component such as beep, or LED at this moment for node's output operation, and can be expended to room light controller, or air conditioner. To increase stability, and reduce the power consumption and size, system needs to be designed in single chip.

\subsection{System Software}

\section{A. The Robotic Soccer Strategy}

The primary characteristic of robotic soccer environment is dynamic and unpredictable mainly due to competitive motion of opponent robots, in which the opponent robots act as dynamic obstacles that have to be avoided. This characteristic of unpredictability makes the control strategy reactive and instantaneous, implying no complete sequence of supervisory control can be assigned in advance. In such a case, an effective state-action model based coordination strategy has been developed which can be considered as a small size simple discrete-event dynamic system(DEDS). The developed model has been successfully applied to Kingo robotic soccer system [11].

\section{B. The Vision Data Processing Algorithm}

The objective of external vision system is to provide visual information of robotic soccer environment including the positions and orientations of team robots and the position of ball, opponent robots, goal post, etc. To this end, an efficient visual processing algorithm developed for middle league Mirosot has been modified and applied to X-large scale robotic soccer system. To reduce the processing time of visual data in finding and identifying objects, the dotted scan-line method were used [10].

\section{The Communication Protocol}

The RF module has been used in transmitting the continuous stream of information data from the centralized coordinator to each robot playing soccer game. In addition, the ultrasonic GPS sensor uses an additional RF module to communicate with other sensors in the sensor network including the mobile robots as active mobile nodes. The information from the central coordinator and address along with synchronizing signals are coded into serial data and sent by the transmitter using a modulating carrier signal of specific frequency. The received serial data are demodulated by the receiver and decoded and interpreted by the microprocessor onboard mobile robot. As for the carrier signals, two separate frequencies, 433 and $418 \mathrm{MHz}$, are prepared for asynchronous serial communication in the robotic soccer game.

\section{The Sensor Node Software}

A compact OS (e.g. tiny-OS in Berkeley Univ.) is embedded within each sensor node. This OS offers common node functions: protocol stack, link management, routing path control, and power management and carry out the assigned sensing tasks in application. Task scheduler manages every resources and running tasks on the node. If a given task requires real-time operation, it should be arranged to perform in extra priority. Power management is another important process in each sensor node. Based on the CPU time of each process collected by power monitor, the power manager determines when the node turns to sleep and resche dule the processes. For effective data exchange, all the tasks share the buffer memory. In exchanging data, data transfer rate is a major factor in network traffic, which in turn is determined by the protocol used and protocol stack management.

\subsection{The Integrated Intelligent Control Architecture} Fig4 is the block diagram of proposed control architecture to implement the new control framework of robotic soccer system with a distributed sensor network. With this control architecture, purely reactive control structures as well as fast deliberative control with a small size DEDS model can be effectively implemented producing robust performance in the dynamic environment of robot soccer. Therefore, a hybrid deliberative/reactive control strategy has been applied within the new control framework for real-time multi-robot coordination with the paradigm of distributed sensing and actuation. In the figure, the sensing module consists of internally wired vision sensor and external wireless sensor network accessed via the attached environment memory (NAEM) which functions as an external logical sensor for physical sensor network in environment and situation sensing.

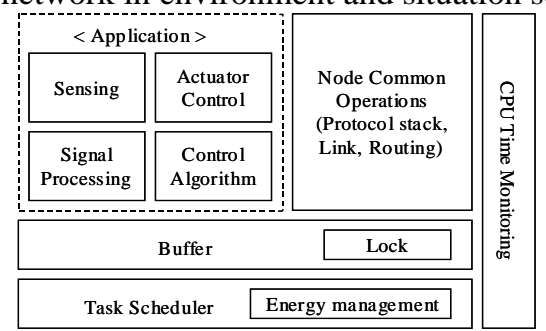

Figure 3. Sensor node software 


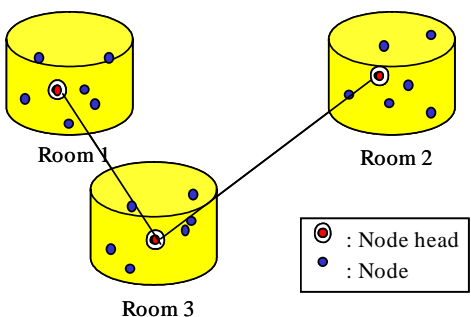

Figure5. Network attached environment memory.

(NAEM) nodes are installed in the workspace: ID 0 is set to cluster head, one mobile robot, and one system monitor used in building up the system. Similarly, each node has been also assigned a unique ID. During the installation of experimental setup, the absolute location of each node has been measured and notified to mobile nodes of robots. In the network, broadcasting, ID based direct calling, and flat routing with fixed routing table has been used to transfer a packet.

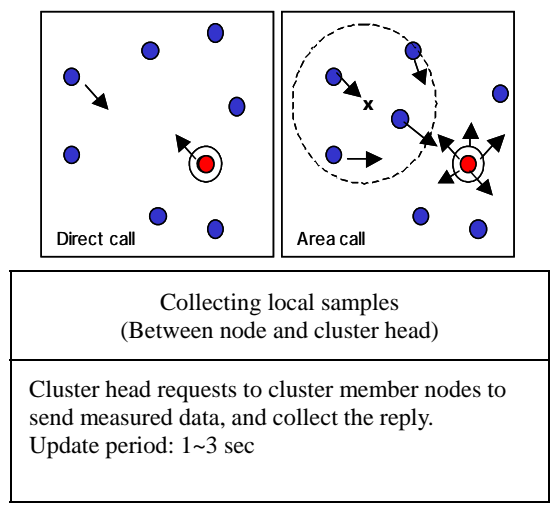

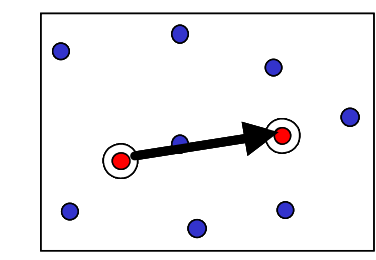

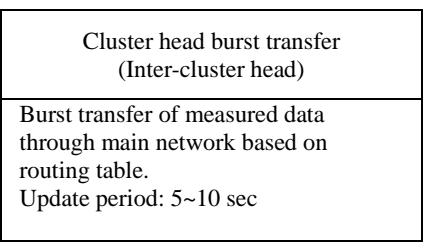

Figure 7. Data transfer types in NAEM

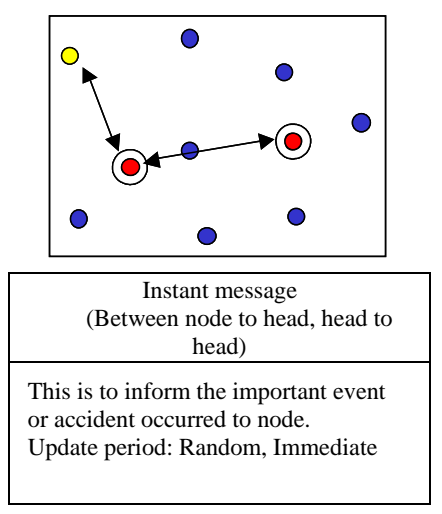

Update period: Random, Immediate
Fig5 shows examples of NAEM in which the sensor nodes with NAEM installed in an interested area or workspace consisting of a cluster and special storage. Each cluster head periodically collects processed sensor data from the cluster member nodes and keeps monitoring the room status stored into internal storage. This information is sent to system monitor via routing path, or to other node when requested. Therefore, as shown in Fig.6, this architecture with NAEM offers fast response rather than accessing to individual nodes to find out the situation when the robot comes to new working area.

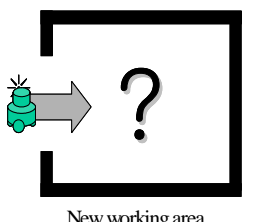

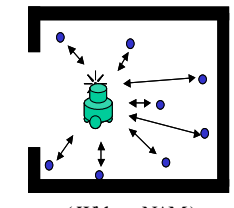

( Without NAM)

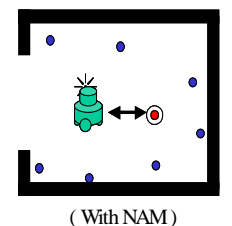

( With NAM)
Figure 6. Robot's situation sensing and NAEM.

Fig.7 shows three types of data transfer in NAEM: collecting local samples, cluster head burst transfer, and instant message. Each cluster head collects measured samples from local node and updates internal memory. Cluster head burst transfer is to send situation data to system monitor or mobile node of robot, which can assign an extra RF channel for reliable communication with collision free from collecting local samples. Instant message of a node is to notice an event to system monitor. This packet has the highest priority and can block other messages.

\section{EXPERIMENT}

The proposed control framework has been implemented and applied to a robot soccer system. In the experiment, we focused on the cooperative behaviour generation of multiple mobile robots to achieve the desired goal. Total 6 of ultrasonic sensor
Each sensor node adopts a compact processor ATMega128 microcontroller from ATMEL. Ultrasonic receiver, PIR sensor, light and temperature sensors are attached, and Radiometrix BIM 433Mhz FM transceiver for wireless communication. Every node has also external power supply installed. As an active moving node, each mobile robot has on top two components: sensor network interface and motion control processor. The hardware configuration of sensor network interface installed on the top of the robot is similar to the one used in the ordinary fixed sensor nodes. The mobile node executes its received command and carries out self-localization of position and orientation with the aid sensor network and its own digital magnetic compass. That is, in determining its own configuration, the mobile robot uses triangular positioning from by measured distance to nearby ultrasonic nodes and measured the directional data from digital magnetic compass. The configuration of each mobile robot is also calculated from the external vision processing algorithm which can be used as a reference value and fused in the sensor network when necessary. During the sampling periods of external sensors, motion control of each mobile robot executes local dead reckoning and feedback control loop in the Intel 80C296 CPU. In addition, the inverse kinematics is necessary in this execution level to carry out the behaviours commanded from the higher level controller, e.g., the supervisory command such as 'move to desired goal'. As for communication protocol of RF module, a custom defined packet shown in Fig. 8 is used for command transmission in the robot soccer system. While the request of packet retransmission or error recovery has not been defined for simplicity, the carrier detection based collision avoidance has been used.

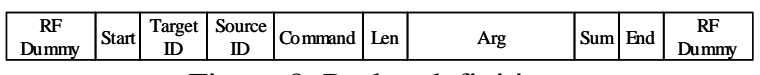

Figure 8. Packet definition. 
Fig. 9 shows the fixed sensor nodes and active mobile node of micro-robot. Fig. 10 shows robot control and network monitoring program written in National Instrument LabVIEW. Status information monitored from Fig. 10 includes robot's dead reckoning posture, measured angle by digital magnetic compass, measured sensor data in each node, and position information from triangular method. In the control and monitoring program, a user command can be transferred such as 'go to an absolute position', 'go to a relative position', and 'set position', through the network. Fig. 11 shows the experimental setup of robotic soccer system. Since a single ultrasonic GPS node installed at $3 \mathrm{~m}$ height can cover more than $3 \mathrm{~m} 2$, six sensor nodes are installed for $440 \mathrm{~m}$ x $280 \mathrm{~m}$ workspace providing high redundancy by permitting partial intersection of the effective coverage areas of adjacent sensor nodes. This means that there is no dead zone in the work space from the reaches of ultrasonic GPS sensor nodes. Initial calibration has been accomplished by the least square algorithm using real distance and measured value of ultrasonic GPS sensors.

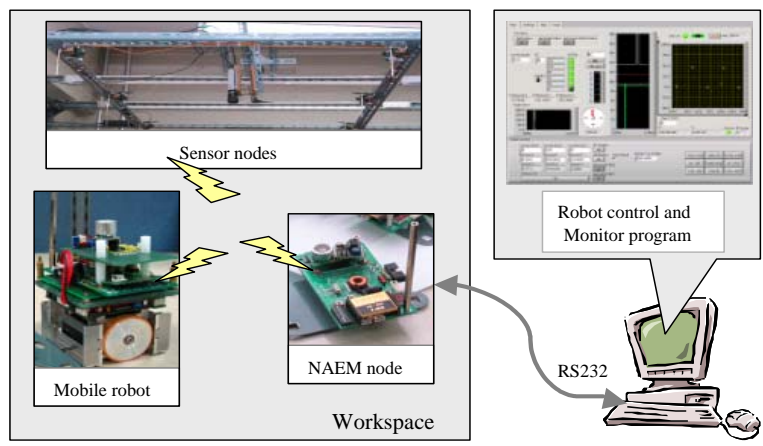

Figure 9. Sensor nodes

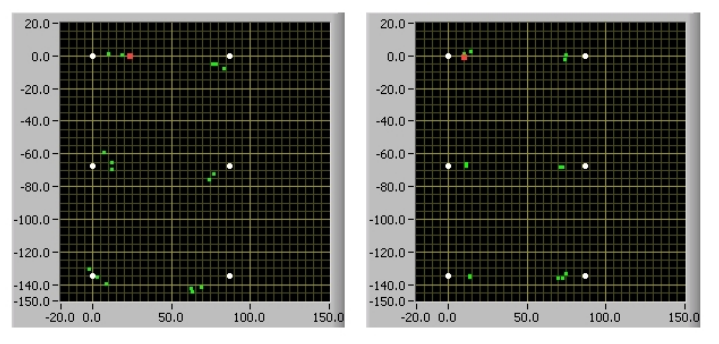

Figure 10. Monitering Program

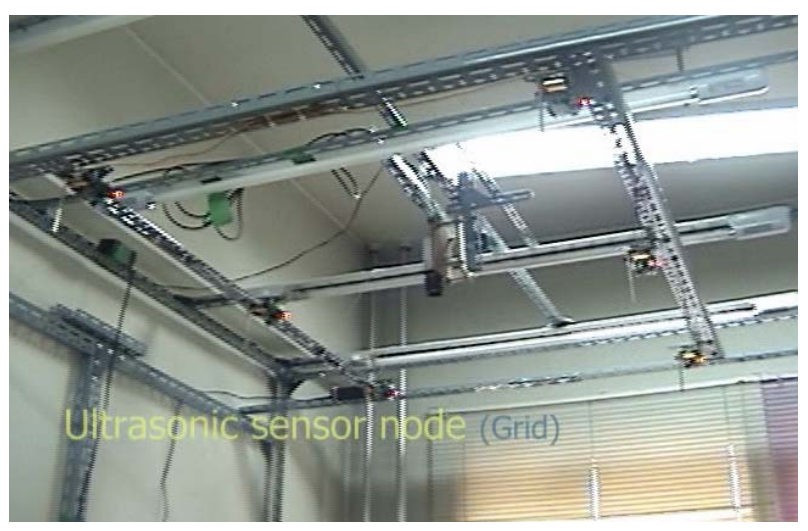

Figure 11. Experimental Setup

\section{CONCLUSION}

This paper presents a new control framework for Xlarge scale robotic soccer system. In the framework, integrated intelligent control architecture is proposed for distributed sensing and control, which accommodates vision sensor, ultrasonic GPS sensors in the sensor network, digital magnetic compass, and optical encoders. In the proposed control architecture, the sensor network with NAEM gives fast data transmission and real-time situation sensing for coordinated motion control of multiple mobile robots. It is demonstrated that even though each sensor node has a limited capability with a simple signalprocessing engine, a group of sensor nodes makes it possible that the new control framework performs a large task of cooperative behavior generation for robotic soccer system through coordinated information sharing and wireless communication.

\section{REFERENCES}

[1] D. Estrin, D. Culler, K. Pister, and G. Sukhatme, "Connecting the Physical World with Pervasive Networks”, IEEE Pervasive Computing, pp. 59-69, January-March 2002

[2] Akyildiz, I. F., Su, W., Sankarasubramaniam, Y., and Cayirci, E., “A Survey on Sensor Networks”, IEEE Communications Magazine, 2002.

[3] Chien-Chung Shen, Chavalit Srisathapornphat, and Chaiporn Jaikaeo. "Sensor information networking architecture and applications”, IEEE Personal Communications, August 2001.

[4] Roy Want and Trevor Pering, Gaetano Borriello, Keith I.Farkas "Disappearing Hardware” IEEE Pervasive Computing, pp.36-47, IRS-TR-02-001, Jan. , 2002

[5] G. J. Pottie and W. J. Kaiser. "Wireless integrated network sensors”, CACM, 43(5):51-58, may 2000.

[6] James WeatherAll. Cambridge University, Alan Jones. "Ubiquitous Network and Their Applications" AT\&T Laboratories Cambridge

[7] N. Priyantha, A. Miu, H. Balakrishman, and S. Teller. "The Cricket Compass for Context-Aware Mobile Applications”. In Proc. of the 7th Annual ACM/IEEE MOBICOM 2000.

[8] Zhao Feng-Ji; Guo Hai-Jiao; Abe, K, "A mobile robot localization using ultrasonic sensors in indoor environment", Proceedings, 6th IEEE International Workshop on , 1997 Page(s): 52 -57

[9] Tae-Kyung Moon, Tae-Yong Kuc "Sensor Network based Localization and Navigation of Mobile Robot” ICCAS October

[10] Woong-Gie Han, Seung-Min Baek and TaeYong Kuc, "Path planning of visual-served multiple mobile robots using the genetic algorithms", Proceedings 96 Micro-Robot World Cup Soccer Tournament, pp. 57-63, November, 1996 [11] Dong-Hun Lee, Seung-Min Baek, Tae-Yong Kuc and Chae-Wook Chung, "Intelligent Hybrid Modular Architecture for Multi Agent System”, ICCAS2004, August.

[12] http://www.fira.net

[13] http://www.kinggorobot.com

\section{ACKNOWLEDGMENTS}

This research was supported by University IT Research Center Project. 